\title{
The molecular and cellular basis of iron toxicity in Iron Overload (IO) disorders. Diagnostic and therapeutic approaches
}

\author{
Zvi loav Cabantchik, ${ }^{1}$ Yan Sung Sohn, ${ }^{1}$ William Breuer ${ }^{1}$, Breno Pannia Espósito \\ ${ }^{1}$ Institute of Life Sciences, Hebrew University of Jerusalem, Safra Campus at Givat Ram; \\ ${ }^{2}$ Israel and Instituto de Química, Universidade de São Paulo, São Paulo SP, Brasil
}

\section{Introduction}

Abnormal iron accumulation in human tissues and oxidative damage are emerging issues in the medical field $(1,2)$. The most commonly recognized type of pathological accumulation has been associated with the general appearance of plasma non-transferrin bound iron (NTBI) (3-16) and particularly with a labile iron component that can infiltrate cells in an unregulated manner (17-19). A major consequence of excess iron accumulation is a rise in cellular labile iron (LCI) $(4,8,10)$ that can promote the formation of reactive oxygenspecies (ROS) from physiological oxygenintermediates (ROI), overriding the cellular antioxidant machineries and causing oxidative damage $(2,8,9,20)$. The major diseases caused by excess iron accumulation are those that result from tissue exposure to systemic rises in plasma NTBI, as in transfusional siderosis (sickle cell anemia, thalassemia major and some forms of myelodysplasia) or primary hemochromatosis and thalassemia intermedia $(1,2)$. However, it is increasingly recognized that various neurological, hematological and more recently chronic diabetes and metabolic disorders may be caused by regional (cell or organ) rather than systemic iron accumulation $(4,9,10,21)$. The regional iron accumulation results from mutations in genes or from humoral factors that affect particular components of cell iron metabolism leading to intracellular maldistribution of the metal $(4,21)$. Iron accumulation of iatrogenic nature has recently been identified in the spleen, liver and pancreas of iv-iron supplemented CKD patients with chronic anemia (22).

The present review focuses on non-physiological (i.e. anomalous) forms of iron in plasma and cells that have been implicated in tissue iron accumulation and multi-organ iron overload and toxicity. Although those pathological forms have not been fully defined in

Correspondence: Zvi Ioav Cabantchik

(C) Copyright Z.I. Cabantchik et al., 2013

Licensee PAGEPress, Italy

Thalassemia Reports 2013; 3(s1):e3

doi:10.4081/thal.2013.s1.e3

This article is distributed under the terms of the Creative Commons Attribution Noncommercial License (by-nc 3.0) which permits any noncommercial use, distribution, and reproduction in any medium, provided the original author(s) and source are credited.

Parts of this work were presented at the

" 3 rd Pan-European Conference on Haemoglobinopathies and Rare Anaemias", Limassol (Cyprus), 24-26 October 2012. chemical and toxicological terms, they have already been perceived as a major source of iron toxicity in systemic iron overload (SIO) disorders $(1,2,6)$. In this review we explore the possibility that those pathological forms of iron might be of clinical value in the diagnosis of impending $\mathrm{IO}$ and in the monitoring of treatment efficacy.

Chemically labile iron as a diagnostic indicator in Systemic Iron Overload (SIO).SIOis a pathological condition characterized by persistently high levels of circulating and organ accumulated iron $(2,6)$. Iron in plasma and interstitial fluids can exceed the effective iron binding capacity of circulating transferrin and generate chemical forms that are not associated with transferrin, hence the term non-transferrin bound iron (NTBI) $(6,14,16,23-25)$. As NTBI has been causativelyimplicated in tissue I0, its level in plasma (or serum)could be perceived as marker/indicator of SIO $(2,9)$ and therefore as a direct target of chelation $(6,9,20,25)$. It would therefore seem appropriate and timely to discuss the potential clinical value of NTBI measurements in I0 disorders, both on its own and in conjunction with the classical serum ferritin, and/or transferrin saturation. In fact, plasma ferritin has for many years served as the major, if not sole, clinical indicator of body iron status, largely because its levels were found empirically to reflect those of iron stored in reticuloendothelial system (RES) and/or liver, which in turn rely on measurements of liver iron concentration (LIC, classically measured in liver biopsies) $(2,9)$. The advent of non-invasive iron scanning devices has gradually allowed tracing organ dysfunctions to excessively accumulated iron not only in liver, but also in heart and endocrine glands (9). The present view is that indicators of tissue IO directly reflect iron that accumulated over extended periods of time, while plasma ferritin reflects iron stores only in liver and spleen, though not uniquely (as also inflammatory conditions accompanied by functional iron deficiency lead to apparently similar changes in serum ferritin). On the other hand, the appearance of plasma NTBI can be perceived as an early indication of SIO per se and consequently of impending organ damage (6). This is because some NTBI forms present in plasma over extended periods of time can infiltrate cells where they may become chemically active and catalyze the generation of ROS that override cellular antioxidant capacities and thereby affect vital cell functions. These properties underlie the rationale of continuous maintenance of NTBI at basal levels as a tangible target in iron chelation therapy (1012,15,26,27).

\section{Non-transferrin-bound iron (NTBI)}

Chemical nature and pathophysiological relevance in SIO.The fact that plasma NTBI is generally detected when spare transferrin binding capacity is diminished would indicate: a. that NTBI is generated at sites where apotransferrin levels might be limiting relative to the plasma infiltrated iron and b. that most/some of the NTBI formed might remain in plasma essentially inaccessible to circulating apotransfer- 
rin. The NTBI that is detected in plasma appears in various chemical forms, depending how much and from where (gut or RES) the excess iron originated, the patient's history of transfusions or phlebotomies (as reflected in the degree of oxidative modifications of circulating proteins), and the ongoing chemical treatments (e.g. chelation). At present it is not clear which is the pathological threshold of NTBI, both as indicator of IO per se and/or as a potential source of cell iron toxicity. Moreover, whether or not the NTBI forms in plasma/interstitial fluids will evoke organ (particularly extra-hepatic) iron accumulation and ensuing damage in thalassemia or hemochromatosis, would depend on three factors: a) the nature and concentration of the permeating species; b) the existence of cell membrane routes through which particular NTBI forms can (opportunistically) gain access into particular cells and c) the time of exposure to those NTBI forms. Given the above factors, the relative contribution of specific routes to IO in particular tissues is likely to vary enormously, compounded by the fact that NTBI can appear as free organic iron complexes $(28,29)$ or complexes that are non-specifically associated with plasma proteins $(7,23,25,29)$. For the free complexes, the relevant plasma NTBI permeating components are either: a. the ionic and free ligand-metal, in which case its ingress might require metal reduction by membrane associated reductases followed by opportunistic translocation of the free-iron via putative transporters or channels (that can handle only free or hydrated divalent ions) or b. organic-iron complex, in which case more complex machineries that can handle iron-ligands should be implicated. If the major permeating species are the protein-bound iron complexes, then their tissue ingress would be limited to bulk endocytotic routes (mostly adsorptive endocytosis) followed by intracellular metal release (19). Clearly, a serial or parallel combination of the above mechanisms, bulk and ionic transport, can possibly account for the handling of all NTBI forms present in the different conditions of IO. However, the relative contribution of particular agencies to NTBI uptake into cells might differ among tissues, depending on the available repertoire of transporters/channels vis àvis the locally available NTBI forms. Possible candidates for NTBI transport are the metal transporter ZIP14, voltage dependent $\mathrm{Ca}$ channels and transporters of organic acids that can also handle iron-chelates, but definitive demonstration is still pending (9).

Definition of NTBI The term NTBI was introduced in order to define non-physiological, low molecular weight forms of iron that appear in plasma or other body fluids of iron overloaded patients and are not tightly associated with transferrin $(14,16)$. The presence of such forms was postulated on the basis that the amount of iron detected in plasma of IO patients often supersedes the plasma transferrin binding capacity (TIBC). However, as the original detection of NTBI in human sera necessitated extraction with mild chelators followed by size filtration $(14,16,23,28,30)$, its native chemical identity in plasma was hampered. Although a major fraction of NTBI is considered redox active $(13,31)$, hitherto, no single component of NTBI has been shown experimentally to be toxic in plasma per se. However, at particular NTBI levels, some components might indeed pose a biological risk as they can slowly infiltrate cells and override their ability to: a) safely absorb the incoming metal and/or b) neutralize their pro-oxidative capacity $(17,19,20,32)$. Thus, although the term NTBI per se is simple and intuitive, it is both too broad and rather ambiguous. For instance, although NTBI by its very nature should comprise also ferritin, whose levels in plasma also rise in pathological SIO (2), it is customary to exclude it irrespective of its iron content. Conversely, chemically stable chelates of iron that are formed during chelation therapy and qualify stricto senso as NTBI (and even measured as NTBI by some assays $(5,23,33,34)$, should be excluded if the chelated iron is not redox active and poses no biological threat. Similarly, synthetic iron-polymer complexes that are administered parenterally, and qualify as NTBI despite being iatrogenic, are not considered bona fide NTBI because they are neither toxic in plasma or to the organism as a whole.
Definition of Labile plasma iron (LPI) and labile cell iron (LCI). Due to the ambiguities inherent in the term NTBI, we opted for designating the potentially toxic forms of iron in biological systems as labile iron, which in plasma we refer to as LPI and in cells as LCI (8-11). The labile iron comprises the redox-active and exchangeable forms of the metal as they appear in their native milieu and, by their very nature, they are also the direct targets of chelators $(10,13)$. In normal physiological conditions, plasma and interstitial fluids are essentially devoid of labile iron (as long as there is sufficient transferrin iron binding capacity) whereas cells maintain a physiological pool of labile iron (LCI), usually at the sub- $\mu \mathrm{M}$ level $(8,10)$ (Figure1). With the appearance of plasma NTBI associated with SIO, there is a concomitant rise in labile plasma iron (LPI) and, in turn, also in LCI, which is derived from long term cell exposure to the former. LCI levels can also change as a result of cell iron maldistribution that is found in pathological conditions associated with inappropriate cell handling of iron (4). The latter is caused by mutations in genes of iron metabolism (e.g. Friedreich ataxia and some forms of sideroblastic anemias) and also by humoral factors that affect iron import/export balance in some cells (e.g. hepcidin on macrophages).

Determination of NTBI. As NTBI in various IO conditions represents less than $1 / 10$ the value of plasma TBI at full saturation $(40-50 \mu \mathrm{M})$, its detection poses some analytical challenges. NTBI appears in plasma bound to small or oligomeric organic ligands (such as citrate) which, in turn, might be either freely filterable or adsorbed to proteins $(25,28,29)$. This heterogeneity forced analytical assays to incorporate extraction/mobilization and filtration steps for complete determination of NTBI, while sparing TBI $(14,16,23)$ (Figure 2). In the clinical setting, the chemical analysis of NTBI is generally preceded by freezing, storing and thawing of sera (or heparinized plasma), steps that ideally should preserve the native TBI and NTBI components. Although most available analytical methods are sensitive and reliable (particularly when no iron chelates are present in patient's plasma), they are relatively laborious and not suited for the clinical setting.

LPI (labile plasma iron) and DCI (directly chelatable iron) as redoxactive and chelatable forms of NTBI $(5-8,13)$ The type of plasma NTBI that is potentially toxic to cells is comprised of iron that can be deposited on cell surfaces as redox-active species and/or excessively taken up by cells in forms that can raise the labile cell iron (LCI). As labile forms are also potentially chelatable, they represent the primary pharmacological target of any chelation treatment aimed at preventing undesirable iron overloading of cells. Unlike the NTBI assays that were

\section{LABILE CELL IRON (LCI)}

physiology and pathology

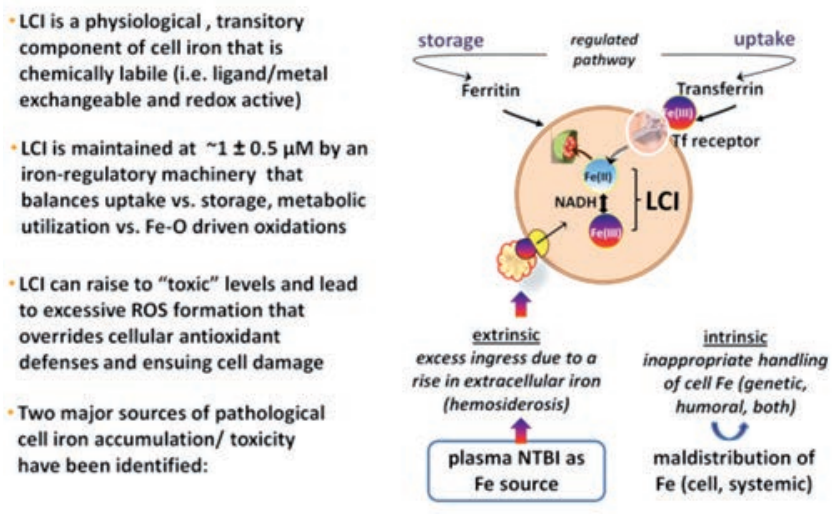

Figure 1. 
designed to reveal all forms of plasma NTBI using extraction-filtration procedures, the LPI and DCI assays (Figure 3)were designed to reveal labile and/or chelatable forms of iron in native biofluids while avoiding potential complications associated with addition of very high concentrations of iron-mobilizing agents.

The LPI assay $(13,27,35)$ detects the ability of iron in plasma (or any other fluid, extra- or intra-cellular) to be prompted by physiological concentrations of ascorbic acid and catalytically oxidize the non-fluorescent dihydrorhodamine (DHR) into the fluorescent rhodamine (R)(Figure 3). The detected change in fluorescence of DHR prompted by ascorbate is attributed to labile iron insofar as the process is depressed or eliminated by a specific iron chelator which is added to the system in a parallel reaction.

The DCI assay (5) detects labile iron with a fluorescent chelator (free or coupled to a polymeric bead) that binds the metal with high affinity and stoichiometrically undergoes a commensurate quenching of the fluorescence (Figure 3). As with the LPI assay, the excess addition of a chelator (with no tag) provides the means to ascertain that the changes in fluorescence can be attributed solely to labile iron and are quantifiable with appropriate iron standards. Changes in fluorescence in biological samples can be followed in spectrofluorimeters or fluorescence plate readers and also in flow cytometers (or FACS machines) with the aid of probes coupled to beads.

Both LPI and DCI are applied as high throughput assays in formats based on fluorescence plate readers. As these assays use no exogenous mobilizing/extraction agent, some (cryptic) NTBI forms might not be detected due to being bound/adsorbed to proteins and/or of low redoxactivity and/or limited accessibility to the iron-detector agents (7). Those cryptic forms can be revealed with the aid of agents that gently mobilize adsorbed NTBI labile species while minimally interfering with plasma components (Figure 3) (7). That was found necessary for detecting NTBI in highly oxidized plasma of hypetransfused-nonchelated thalassemia patients (7), but its pathophysiological significance is unclear. We found that the inclusion of moderate concentrations of a mild mobilizer (e.g. 0.1-0.5 mM nitrilotriacetic acid, NTA) not only increased the sensitivity of LPI or DCI assays but also reduced possible cumulative interferences by albumin, citrate and uric acid in different patient samples (7).

Importantly, the adopted modification caused no release of iron from fully saturated transferrin (TBI) or iron chelates like deferrioxamine (DF0), nor from the relatively weaker chelators deferiprone (DFP) or deferasirox) (DFR) (7). The original and the modified methods, namely without or with the mobilizing agent, are referred as LPI and eLPI ('e' for enhanced or extracted LPI) and DCI and eDCI (enhanced or extracted DCI). Both eLPI and eDCI were highly correlated with each other and are perceived essentially as equivalent to NTBI (7).

Adaptation of DCI and LPI measurements to flow cytometry.A prototype method was developed in order to expand the scope of applications of DCI measurements and render the latter more accessible to clinical laboratories where flow cytometers (FACS technology) are essential instrumentation. The principle is essentially based on recruiting chelatable iron from biofluids via microsphere beads coated uniformly with a fluorescence metal sensor that senses the metal while binding to it with high affinity from diluted samples. The measurement of the bead-associated fluorescence in a flow cytometer (or equivalent instrument-FACS: fluorescence activated cell sorter) permits the assessment of changes in signal intensity that reflect binding of chelatable iron. Besides the sensitivity of the method which is comparable to DCI and LPI, it is less susceptible to interferences by medium components, allowing its application to plasma and serum alike, urine, cerebrospinal fluid, body exudates, saliva, etc. The bead technology has two modalities that cover:

Measurement of DCI in fluids with the aid of beads and flow cytometry (DCIB) (36) (Figure 4). The DCIB probes are microspheres or beads of defined size that are constructed of derivatized polystyrene onto which a dendrimer derivatized with both the fluorophore fluorescein (FI) or rhodamine (R) and the chelator DF0 are coupled covalently by a cross-linker. The resulting fluorescent DFO beads (FDB for fluorescein +DFO and RDB for rhodamine+ DFO beads) are calibrated for iron sensitivity with solutions containing iron saturated plasma/serum or urine or simple iron standards in buffered saline solution. The changes in fluorescence are attributed specifically to iron based on the

Measuring plasma NTBI (total)

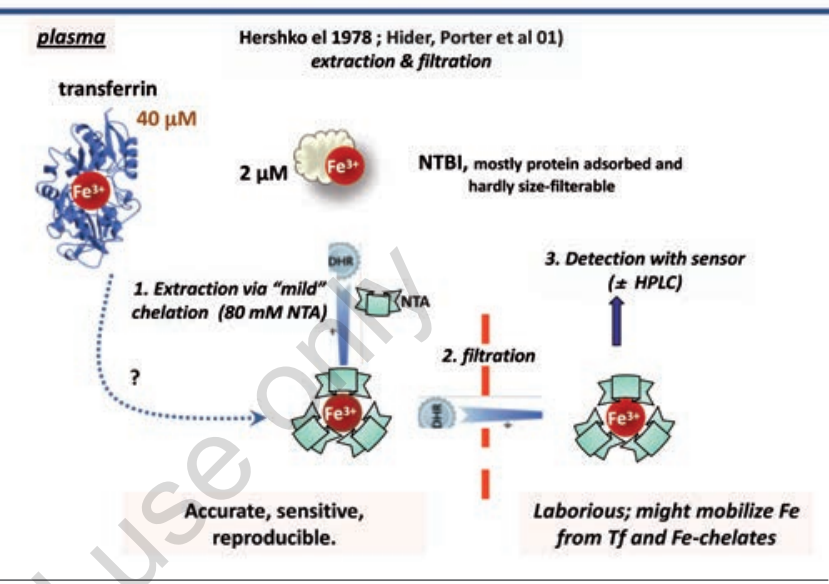

Figure 2 .

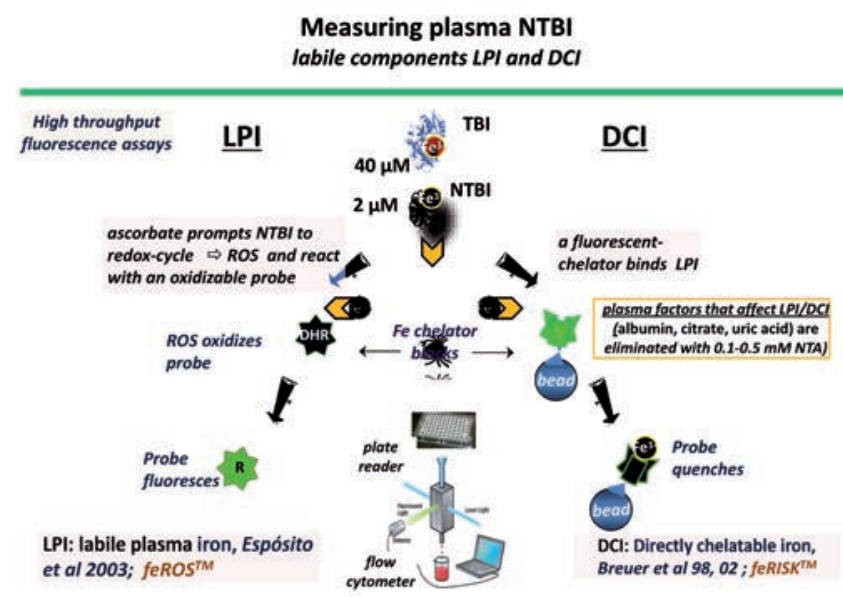

LPI and NTBI levels in Patients with Iron Overload

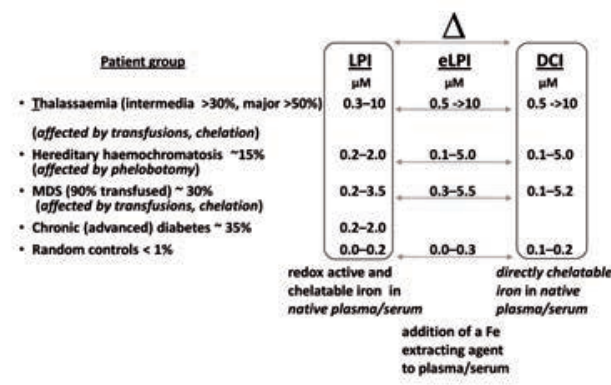

Figure 3 . 
fact that addition of excess amount of the iron chelator deferrioxamine (DFO) to samples essentially eliminates the contribution of the metal. Thus only the DFO-preventable change in signal is taken as equivalent to the presence of a given concentration of iron. A minimal concentration of mobilizing agent (NTA $0.1 \mathrm{mM}$ ) might be used to increase somewhat the sensitivity of the assay by extracting iron adsorbed to proteins or citrate present in samples.

Measurement of LCI levels in living cells by flow cytometry $(18,32,37,38)$ (Figure 5). The labile cell iron content of cells (LCI) represents transitory forms of the metal that are redox active, exchangeable and chelatable and therefore are the most pertinent physiological- ly, pharmacologically and toxicologically $(8,10)$. LCI is typically determined in living cells with the probe calcein (green CALG or blue CALB) which is loaded into cells via permeant acetomethoxy-precusors CALGAM or CALB-AM. The intracellularly released (and trapped) probe interacts with resident labile iron and undergoes quenching commensurate with LCI, which in turn is revealed by addition of a permeant iron chelator, as exemplified in Figure 5 for K562 cells. The green fluorescent CALG generated intracellularly from CALG-AM fills the cytosolic and nuclear space of cells and upon addition of permeant chelator (SIH or DFP) there is a rise in fluorescence F corresponding to CALG-Fe released by the chelator. With the aid of appropriate calibration curves,

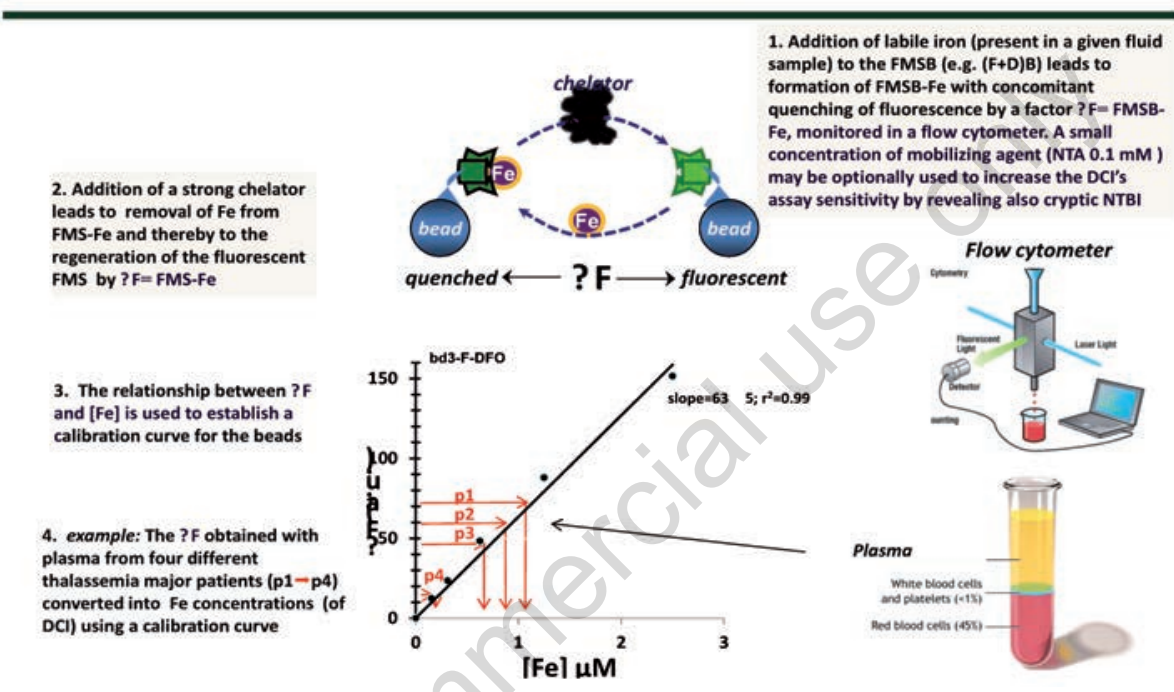

Fluorescence metal sensors on beads (FMSB) as probes for labile iron Measurement of plasma NTBI as directly chelatable iron (DCI) by flow cytometry (FC)

Figure 4.

\section{Assessing labile cell iron (LCI) by microscopy and flow cytometry}

Brever, Epsztejn and Cabantchik, 95-98; Prus and Fibach 2008; Shvartsman, Fibach and Cabantchik, 2010.

CALG as probe for monitoring $L C I$ as directily chelatable iron (DCI)

\section{microscopy}

CALG is added as non-fluorescent CALG-AM precursor that permeates into cells and releases fluorescent CALG that binds labile cell iron LC

and undergoes quenching
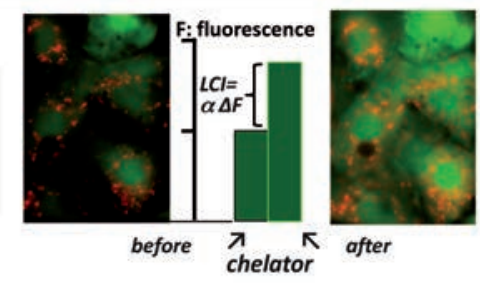

\section{Addition of a strong permeant chelator removes Fe from CALG-Fe, causing cell fluorescence $F$ to rise, so that $\Delta F=[C A L G-F e] \alpha L C I$}

\section{flow cytometry}

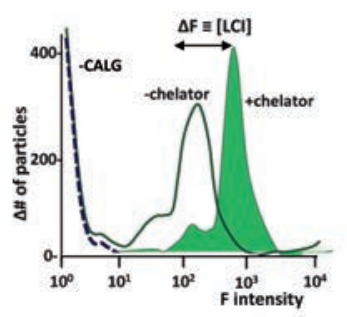

The shifts in CALG fluorescence $\Delta F$ can be converted into LCI conc. with calibrating beads

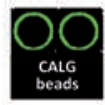

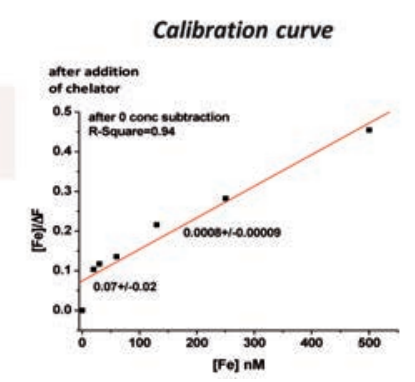

Fig. 5 (bottom right)Measurement of LCI by flow cytometry. The upper figure depicts the labeling of K562 erythroleukemia cells with calcein-green (CALG) via its acetomethoxy AM precursor before and after addition of the chelator $(100 \mu \mathrm{M}$ DFP). The lower left panel depicts the fluorescence distributions of the cells obtained in a flow cytometry before and after addition of DFP. The change in fluorescence elicited by the cell permeant chelator $\Delta \mathrm{F}$ provides a measure of LCI. The lower right graph provides the calibration curve for converting $\Delta \mathrm{F}$ to LCI by using CALG-beads and titrating them with known amounts of Fe. A plot of the weighted reciprocal change in fluorescence $[\mathrm{Fe}] /[\Delta \mathrm{F}] \mathrm{vs}[\mathrm{Fe}]$ was linear for a wide range of $[\mathrm{Fe}]$, allowing conversion of shifts in fluorescence of CALladen cells elicited by chelators $(\Delta F)$ into concentrations $(\mu \mathrm{M})$, as described elsewhere(8). 
LCI can be calculated from $\Delta \mathrm{F}$, as described elsewhere (8). The analogous measurement of LCI can be done by flow cytometry, by estimating $\Delta \mathrm{F}$ from of the mean fluorescence intensity (FL1-H) values before and after the addition of the permeant chelator SIH. The mean change in fluorescence intensity elicited by a permeant chelator (SIH or DFP) on blood samples containing erythrocytes and reticulocytes preloaded with CAL-AM is obtained from the difference in median intensity values (from plots as those shown above for fluorescence associated with each cell population determined with appropriate instrument settings, lower panel). For flow cytometry, the conversion of $\Delta \mathrm{F}$ into actual LCI concentrations necessitates calibration with CALG-coated beads that are titrated with known amounts of iron. Those in turn are measured in the flow cytometer before and after addition of a strong chelator that can displace the CALG-bead bound iron.

Examples of applications of LPI and DCI in clinical trials of different chelators used for following treatment of thalassemia intermedia (Figure 6) and major (Figure 7) and myelodysplastic syndrome (MDS) (Figure 8). For the treatment of hemochromatosis by phlebotomy see Ref. 9

LPI in thalassemia intermedia ( $\beta$-thal/HbE) patients(Figure 6). Correlation between LPI and classical parameters of IO in pre-chelated patients (upper Figure) and in the course of treatment with deferiprone (DFP).

The upper figure shows how LPI levels determined prior to initiation of treatment are correlated with those of serum ferritin (SF), transferrin (Tf) saturation and red blood cell membrane ( $\mathrm{RBCm}$ ) iron. A study published in preliminary form (39) showed a strong correlation between LPI and LCI (measured by R2* MRI) in thalassemia major patients $(n=83)$ following different chelation protocols.

The lower left figure shows the \% change in blood parameters following 16 months of treatment (filled bars). The numbers in the bars indicate the number of months required for attaining a reduction of $50 \%$ in the indicated parameter. The lower right figure shows the kinetics of

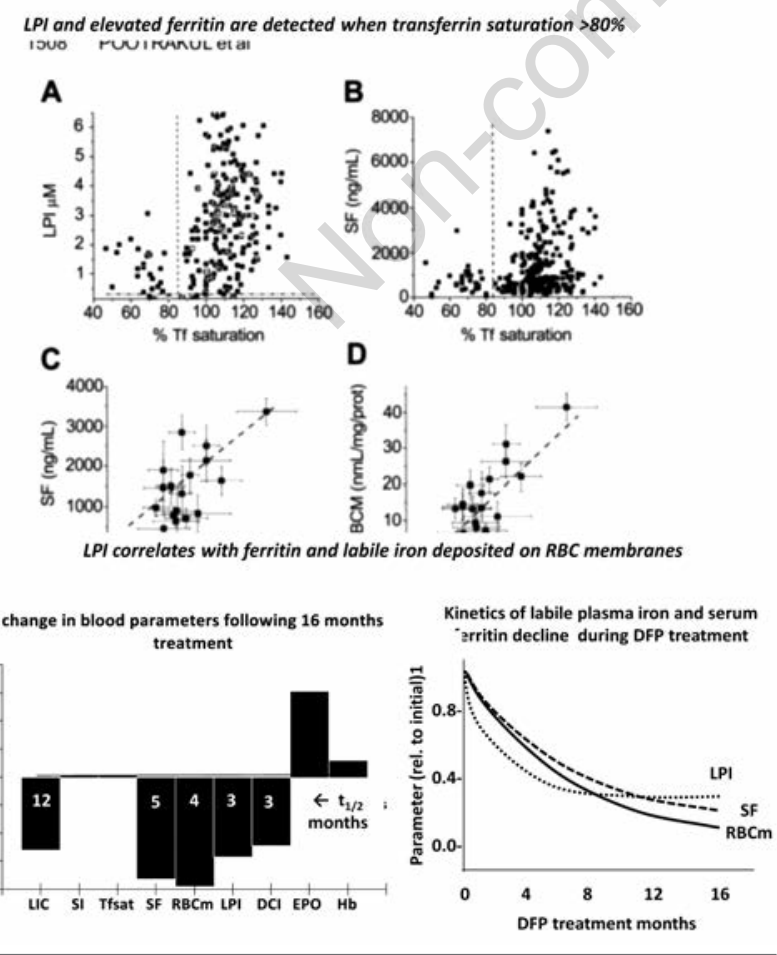

Figure 6.
LPI, SF (serum ferritin) and RBCm (red blood cell membrane iron) and $\%$ transferrin saturation (all in relative units) during the course of treatment with DFP (daily a total of $50 \mathrm{mg} / \mathrm{kg}$ in 2 doses $12 \mathrm{~h}$ apart). The LPI depicts morning trough or basal levels of LPI, following the longest daily period following intake of chelator. LPI essentially declined fastest, followed by RBCm and SF.

LPI in $\beta$-thalassemia major (Figure 7). Daily and intermittent follow up of patients treated with different chelators (compiled and modified from Ref. 12 and 19). LPI in thalassemia major patients was followed hourly during an entire day of chelation with various chelators (upper Figure) and a different group of patients treated daily with DFR and analyzed every 4-6 weeks at trough levels of DFR following (Figure 7 lower panel).

\section{Conclusions}

From a pathophysiological standpoint, LPI represents the major component in plasma causatively associated with tissue iron accumulation and ensuing overload. LCI in circulating blood cells, while not directly affected by I0, provides a measure for the amount of cell accumulated iron due to prolonged exposure to LPI. A major aspect of IO addressed in this review was the evaluation of LPI and LCI in circulating blood cells as merely another indication for overt IOor whether their analysis could providean early indication: a) diagnostically, for impending/emerging IO and b) therapeutically, for assessing chelation efficacy. First and foremost, we emphasized that LPI and LCI should be viewed as dynamic parametersthat are affected, respectively by: a) the outpour of iron into plasma or infiltration into cells (inherently related to the disease or of iatrogenic origin- as iv iron supplementation) and b) their utilization (natural or induced) or their neutralization by natural components,added chelators or venesecting therapies. Thus unlike
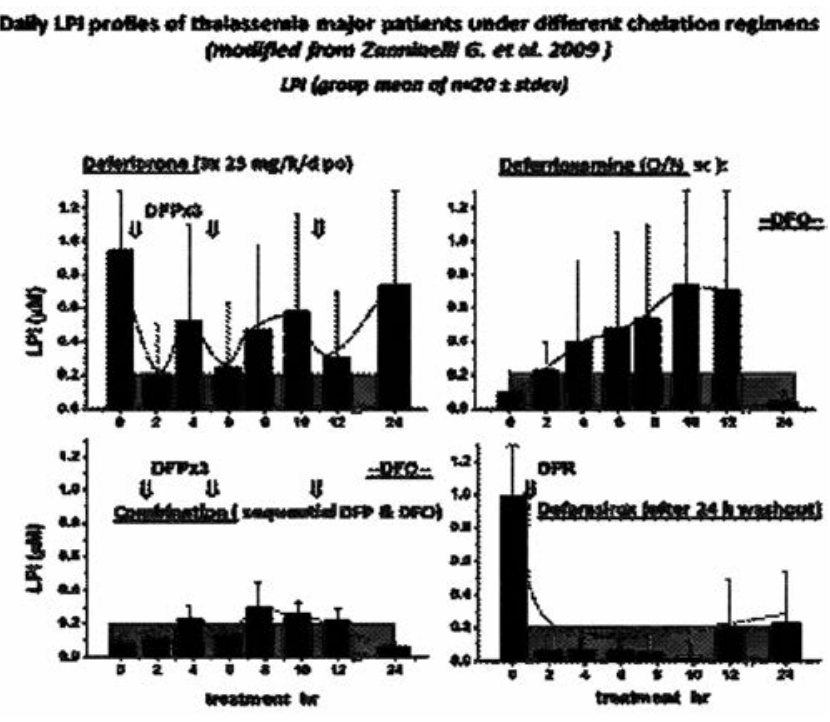

Figure 7. 
ferritin that in serum (in the absence of inflammation) provides a systemic measure for long term iron accumulation/depletionin the RES (liver and spleen), LPI provides an instantaneous measure of labile iron in plasma and LCI in circulating blood cells.

In summary, we propose the measurement of LPI as follows:

- diagnostically (for assessing overt SIO), after a suitable washout period (from chelator or venesection, applicable);

- therapeutically(for assessing chelation efficacy), in two modalities: a) as single LPI measurement taken at trough chelator levels in plasma ( $\sim 24 \mathrm{~h}$ after administration of DFR, 10-12 $\mathrm{h}$ of DFP and 12-14 $\mathrm{h}$ of DF0); this provides a measure for the ability to maintain LPI at basal levels $(<0.2 \pm 0.1 \mu \mathrm{M})$ at a given day in the course of chelation treatment of thalassemia major, thalassemia intermedia, SCD or MDS patients or a regimen of venesection applied to hemochromatosis patients.

- as repeated (bimonthly) LPI measurements at trough chelator levels in the course of treatment as indication for attainment of basal levels as therapeutic target.

It was found that adherence to a treatment that maintains LPI at

\begin{abstract}
Deferasirox on low-/Int-1-risk MDS patients prospective assessment of time required to attain basal levels modified Greenberg P. et al. 2010
\end{abstract}
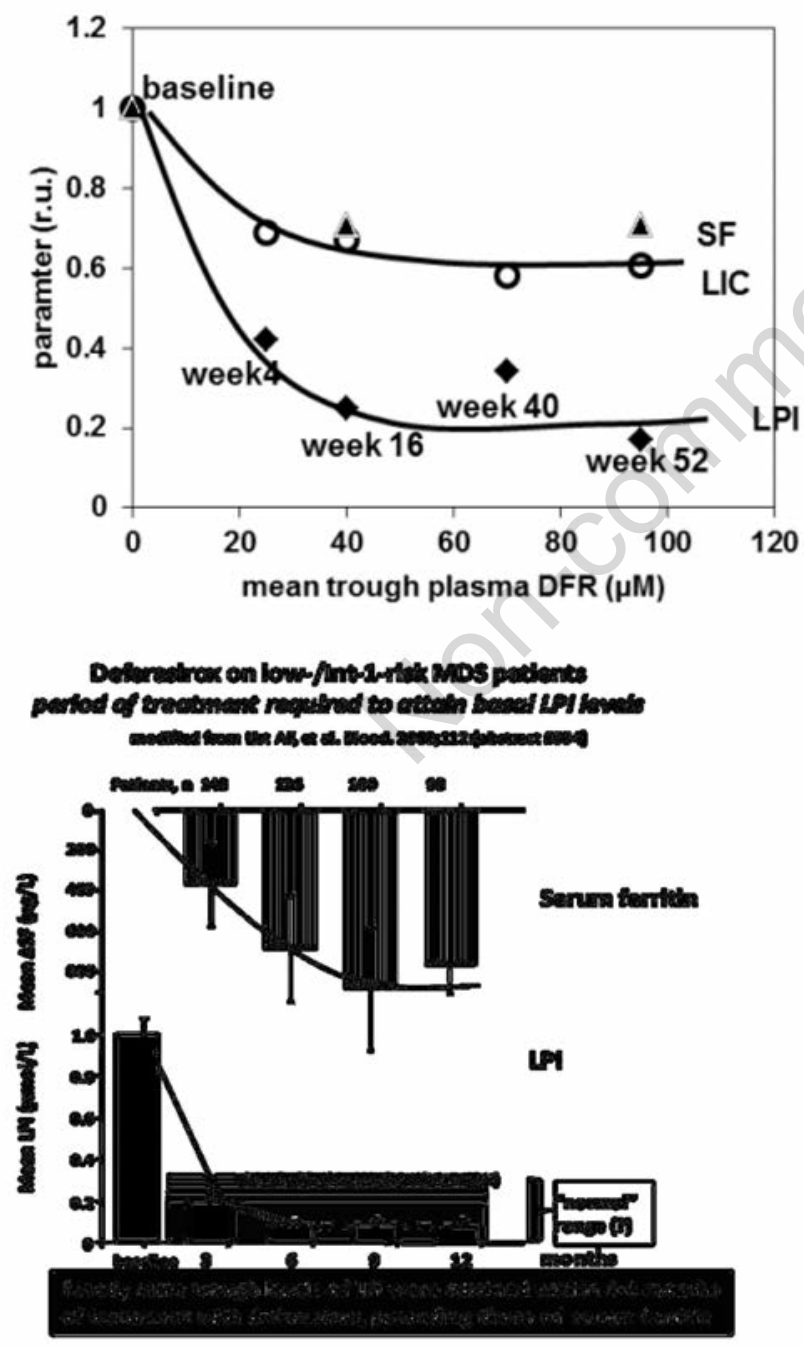

Figure 8. LPI in MDS patients treated with different chelators (compiled from Ref. 15 and 26). basal levels for an extended time period predicts the eventual reduction in liver IO parameters (LIC and SF). Although $>80 \%$ of thalassemia major patients $(n=40)$ with cardiac conditions had LPI $>0.4 \mu \mathrm{M})$, a causative association between cardiomyopathies and exposure to a particular level of LPI/NTBI for a given period time, remains to be established for all forms of systemic IO (persistent plasma Tf sat $>70 \%$ ).

\section{References}

1. Fleming RE, Ponka P. Iron overload in human disease. N Engl J Med 2012; 366:348-59.

2. Porter JB. Pathophysiology of transfusional iron overload: contrasting patterns in thalassemia major and sickle cell disease Hemoglobin 2009;33:S37-S45.

3. Bradley SJ, Gosriwitana I, Srichairatanakool S, et al. Non-transferrin-bound iron induced by myeloablative chemotherapy. Brit $\mathrm{J}$ Haematol 1997; 99:337-343.

4. Breuer W, Cabantchik Z.I. (2009) Disorders affecting iron distribution: causes, consequences and possible treatments, Blood Med.com. http://www.bloodmed.com/800000/mini-reviews1.asp?id $=253 \& \mathrm{p}=1 \& \mathrm{v}=1$

5. Breuer W, Ermers MJ, Pootrakul P, et al. Deferrioxamine-chelatable iron a component of serum non-transferrin-bound iron used for assessing chelation therapy. Blood 2001;97:792-798.

6. Breuer W, Hershko C, Cabantchik ZI. The importance of non-transferrin bound iron in disorders of iron metabolism. Transfus Sci 2000;23:185-191.

7. Breuer, W., Ghoti, H., Shattat, A., Goldfarb, A., Koren, A., Levin, C. Rachmilewitz, E. and Cabantchik, Z.I (2012) Non-transferrin bound iron in Thalassemia: differential detection of redox active forms in children and older patients. Am. J. Hematol. 87:55-61..

8. Breuer, W., Shvartsman, M., Cabantchik, Z.I. (2007) Intracellular labile iron. A review. Int J Biochem Cell Biol. 40: 350-354

9. Brissot P, Ropert M, Le Lan C, Loreal 0. Non-transferrin bound iron: A key role in iron overload and iron toxicity. Biochim Biophys Acta. 2011 Aug 9. [Epub ahead of print]

10. Cabantchik Z.I., Fibach, E. and Bruer, W. 2009. Can labile plasma iron (LPI) and labile cell iron (LCI) levels serve as early indicators of chelation efficacy in iron overload? BloodMed.com. Jul 2009. http://www.bloodmed.com/800000/mini-reviews1.asp?id=254

11. Cabantchik ZI, Breuer W, Zanninelli G, Cianciulli P. LPI-labile plasma iron in iron overload. Best Pract Res Clin Haematol 2005; 18:277-287.

12. Daar S, Pathare A, Nick H, et al. Reduction in labile plasma iron during treatment with deferasirox a once-daily oral iron chelator in heavily iron-overloaded patients with beta-thalassaemia. Eur J Haematol 2009;82:454-457.

13. Esposito BP, Breuer W, Sirankapracha P, et al. Labile plasma iron in iron overload: redox activity and susceptibility to chelation. Blood $2003 ; 102: 2670-2677$.

14. Graham G, Bates GW, Rachmilewitz EA, Hershko C. Nonspecific serum iron in thalassemia: quantitation and chemical reactivity. Am J Hematol 1979;6:207-217.

15. Greenberg PL, Koller CA, Cabantchik ZI, et al. Prospective Assessment of Effects on Iron Overload Parameters of Deferasirox Therapy in Patients with Myelodysplastic Syndromes. Leuk Res 2010;34:1560-5.

16. Hershko C, Graham G, Bates GW, Rachmilewitz EA. Non-specific serum iron in thalassaemia: an abnormal serum iron fraction of potential toxicity. Brit J Haematol 1978;40:255-263.

17. Glickstein, H, Ben El, R., Shvartsman M. and and Z. Ioav 
Cabantchik. Intracellular labile iron pools as direct targets of iron chelators. Blood. 2005 106: 3242-3250

18. Shvartsman M, Kikkeri, A, Shanzer A. and Cabantchik ZI. Iron accesses mitochondria from a cytosolic pool of non-labile iron. Biological and clinical implications. Am J Physiol Cell Physiol 2007 293: C1383-C1394

19. Sohn, Y.S., Ghoti, H., Breuer, W., Rachmilewitz, E.A., Attar, S, Weiss, G. and Cabantchik, Z.I. The role of endocytic pathways in cellular uptake of plasma non-transferrin iron. Haematologica Published Ahead of Print on December 16, 2012, as doi:10.3324/haematol.

20. Glickstein H, Ben-El R, Link G, et al. Action of chelators in ironloaded cardiac cells: Accessibility to intracellular labile iron and functional consequences. Blood 2006; 108:3195-3203.

21. Kakhlon, 0., Breuer, W, Munnich, A. and Cabantchik ZI.. Iron redistribution as a therapeutic strategy for treating diseases of localized iron accumulation. Can. J. Physiol. Pharmacol. 2010 88:187-196 (review).

22. Ghoti H, Rachmilewitz EA, Simon-Lopez R, Gaber R, Katzir Z, Konen E, Kushnir T, Girelli D, Campostrini N, Fibach E, Goitein 0. Evidence for tissue iron overload in long-term hemodialysis patients and the impact of withdrawing parenteral iron. Eur $\mathrm{J}$ Haematol. 2012 Jul;89(1):87-93. Singh S, Hider RC, Porter JB. A direct method for quantification of non-transferrin-bound iron. Anal Biochem 1990;186:320-323

23. Gosriwatana I, Loreal 0, Lu S, et al. Quantification of non-transferrin-bound iron in the presence of unsaturated transferrin. Anal Biochem 1999; 273:212-20.

24. Hershko, C, Link, G, Konijn AM and Cabantchik. Z.I. (2005). Iron Chelation Ther. Curr Hematol. 4:110-116

25. Hider RC, Silva AM, Podinovskaia M, Ma Y. Monitoring the efficiency of iron chelation therapy: the potential of nontransferrin-bound iron. Ann NY Acad Sci 2010;1202:94-99.

26. List AF, Baer MR, Steensma DP, Raza A, Esposito J, Martinez-Lopez $\mathrm{N}$, Paley C, Feigert J, Besa E Deferasirox reduces serum ferritin and labile plasma iron in RBC transfusion-dependent patients with myelodysplastic syndrome J Clin Oncol. 2012 Jun 10;30(17):2134-9. Epub 2012 Apr 30.

27. Zanninelli G, Breuer W, Cabantchik ZI. Daily labile plasma iron as an indicator of chelator activity in Thalassaemia major patients. Brit J Haematol 2009;147:744-751.

28. Evans RW, Rafique R, Zarea A, et al. Nature of non-transferrin- bound iron: studies on iron citrate complexes and thalassemic sera. J Biol Inorg Chem 2008; 13:57-74.

29. Silva AM, Hider RC. Influence of non-enzymatic post-translation modifications on the ability of human serum albumin to bind iron Implications for non-transferrin-bound iron speciation. Biochim Biophys Acta 2009;1794:1449-1458

30. Jacobs EM, Hendriks JC, van Tits BL, et al. Results of an international round robin for the quantification of serum non-transferrinbound iron: Need for defining standardization and a clinically relevant isoform. Anal Biochem 2005;341:241-250.

31. Evans PJ, Halliwell B. Measurement of iron and copper in biological systems: bleomycin and copper-phenanthroline assays. Meth Enzymol 1994;233:82-89.

32. Shvartsman, M., Fibach, E. and Cabantchik, Z.I.. Transferrin-iron routing to the cytosol and mitochondria as studied by live and realtime fluorescence. Biochem. J. 2010 429:185-193.

33. Evans P, Kayyali R, Hider RC, et al. Mechanisms for the shuttling of plasma non-transferrin-bound iron (NTBI) onto deferoxamine by deferiprone. Transl Res 2010;156:55-67.

34. Kolb, AM, Smit NP, Lentz-Ljuboje R, et al. Non-transferrin bound iron measurement is influenced by chelator concentration Anal Biochem 2009;385:13-19.

35. Pootrakul P, Breuer W, Sametband M, et al. Labile plasma iron (LPI) as an indicator of chelatable plasma redox activity in iron overloaded beta-thalassaemia/HbE patients treated with an oral chelator. Blood 2004;104;1504-1510.

36. Sohn, Y.S., Breuer, W., and Cabantchik, Z.I. A microbead based assay of labile iron suitable for flow cytometry analysis. In preparation.

37. Prus E, Fibach E. The labile iron pool in human erythroid cells. $\mathrm{Br}$ J Haematol. 2008 May 22. [Epub ahead of print]

38. Prus E, Fibach E. Flow cytometry measurement of the labile iron pool in human hematopoietic cells. Cytometry 2008 73:22-7

39. Assis, RAA, Esposito, BP, Kay,FU, Baroni, RH, Rosemberg, LA, Nomura, CH, Ribeiro, AAF, Loggetto, SR, Verissimo, MPA, Fabron Jr, A, Araujo, AS, Baldanzi, GR, Nakashima, SS, Diniz, MS, Steagal, M, Velloso, CAGS, Funari, MBG, Wood, J, Hamerschlak, N. The Role of Labile Plasmatic Iron (LPI) In the Assessment of Iron Overload In $\beta$-Thalassemic Patients and Its Correlation with MRI Findings. American Society Hematology Meeting Dec 2010. Poster 2072.

\section{Oral presentation}

Oral presentation is available online 